\title{
PHASE STATES IN POTASSIUM OLEATE+WATER AND POTASSIUM OLEATE+WATER+DECANOL LYOTROPIC SYSTEMS
}

\author{
A. Nesrullajev, N. Kazanci and T. Yildiz \\ Department of Physics, Faculty of Science, Ege University \\ 35100 Bornova-İzmir, Turkey
}

(Received May 19, 1998)

\begin{abstract}
In this work the investigations of the characteristic textures and the dynamics of their concentration and temperature transformations in potassium oleate+water lyotropic system have been carried out. The effect of the decanol on lyotropic mesomorphism as well as on the cliaracter of typical textures and the concentration ranges of lyotropic liquid crystalline mesophases have also been studied. The phase states diagrams of the potassium oleate+water and potassium oleate+decanol+water lyotropic liquid crystalline systems are presented.
\end{abstract}

PACS numbers: 42.70.Df, 82.70.-y, 82.70.Dd

\section{Introduction}

Due to similarity of macro- and microscopic properties of liquid crystals, a certain set of characteristic textures corresponds to each particular type of molecular and micellar structures of lyotropic systems. These textures are sensitive to the change of temperature, concentration, type and the number of the system components [1-6]. Therefore, the stability and the change of the above textures and their related modifications of micellar structures in such systems are of great importance in physics and physical chemistry of liquid crystals.

Of particular interest is the investigation of the effect of different additions on the lyotropic mesomorphism, temperature and concentration regions of lyotropic phases [7-11]. The study of the effect of additional components introduced into the lyotropic system on mesomorphous, morphiological and thermodynamic properties of lyotropic systems are also of special interest.

The investigation of the characteristic textures and the dynamics of their transformations with temperature and concentration variation in amphiphile+water system have been carried out in the present paper. The effect of the third component (decanol) on lyotropic mesomorphism as well as on the character of typical textures and on the concentration ranges of lyotropic mesophases has also been studied. 


\section{Materials and methods}

\subsection{Materials}

An amphiphile substance of potassium oleate (PO) was obtained in the laboratory conditions and triply purified by means of recrystallization. The purity of potassium oleate which was controlled chromotographically was no less than 99.3\%. Decanol (DeOH) was from Merck (cat. no 803463), purity > 99.0 and it was used without further purification. The water was twice distilled with a simultaneous deionization.

In order to prepare the lyotropic systems with corresponding concentrations of components, they were weighted to an accuracy of $\pm 10^{-4} \mathrm{~g}$ with the precision weigher of Einschalen-Feinwagen 707 type. To obtain the homogeneous lyotropic systems, the components were mixed by magnetic mixer and shaker. When necessary, the mixtures placed in a containment vessel were held in a thermostat. The homogeneity of the obtained systems was controlled by a polarizing microscope.

\subsection{Methods}

In the present paper the investigations of the above textures and their modifications were carried out by polarization-optical method. This method which is based on the crystal optics laws is one of the most simple and convenient method for studying the morphological and optical properties of anisotropic media $[12,13]$. Polarization microscopy was widely used for mesophase identification in thermotropic liquid crystals [14-16]. The above method, as applied to lyotropic liquid crystalline systems, has been developed in Refs. [1,17-22] and consists of the following. Due to similarity of micro- and macroscopic properties of liquid crystals, a certain set of characteristic textures corresponds to each particular type of structural ordering. Such a similarity of structures and their corresponding textures were noted yet by Grandjean and Friedel [22, 23]. Thus, when investigating the above textures and peculiarities (degree and the type of orientation, the value and the sign of birefringence, amount and directions of axes of textures, etc.) using the polarizing microscope, one can identify them rather exactly.

\section{Results and discussion}

In the studies of $\mathrm{PO}+$ water.lyotropic system, four types of liquid-crystalline textures corresponding to different amphiphile concentrations were observed. Up to the amphiphile concentration of $25 \%$, the lyotropic system was in the isotropic phase. In this case, the field of vision of microscope with the crossed polarizers remained dark. At amphiphile concentrations from 25 to $50 \%$, a banded (streaky) texture representing a system of separate birefringent formations with a different micellar orientation was observed (Fig. 1a). Such a texture can be obtained from a fan-like texture due to a shift of supporting surfaces of a plane capillary that indicates the hexagonal mesophase $E$. The texture considered was stable and remained constant as long as possible. In the range of amphiphile concentrations from 55 to $65 \%$ another texture was observed (Fig. 1b). In this case, the separate polycrystalline fibres with the length of $80 \mu \mathrm{m}$, not bonded to one another, were 


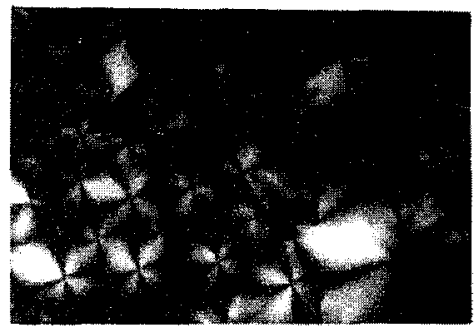

(a)



(c)

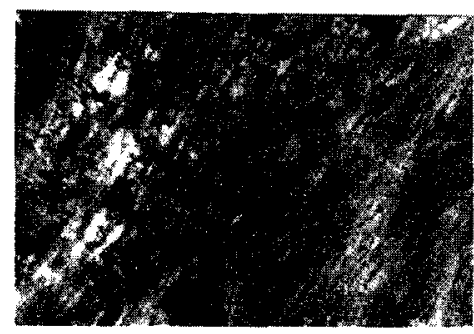

(b)

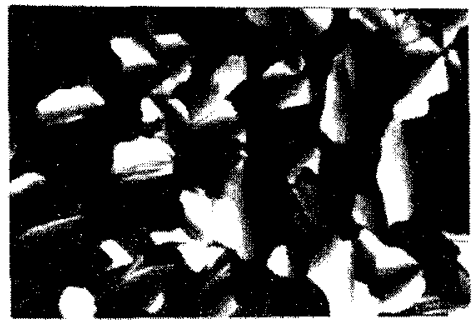

(a)

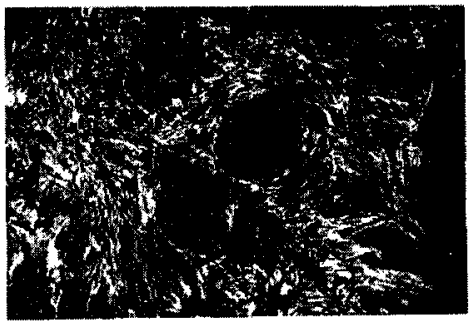

(e)

Fig. 1. Textures of potassium oleate+water lyotropic system: (a) texture of simple hexagonal mesophase $E$, (b) texture of rectangular mesophase $R$, (c) texture of complex hexagonal mesophase $H_{\mathrm{c}}$, (d) texture of lamellar mesophase $D$, (e) texture of gel state.

distributed on the isotropic background with a high density. When the amphiphile concentration was $65-69 \%$, the third type of liquid crystalline textures, i.e., polycrystalline and with a high birefringence, was revealed (Fig. 1c). With amphiphile concentrations from 69 to $90 \%$ and rèlatively high temperatures, a fan-like texture was observed. When the sample was heated to the isotropic liquid state with a subsequent cooling, separate formations, whose density was increased with decreasing temperature up to the mesophase state, were observed on the isotropic background, as the sample was cooled (Fig. 1d). As is known [1, 24], these formations are the evidences of lamellar packing of molecules, and hence, their appearance in concentration region points to the layer structure of the mesophase considered. At certain temperatures, different for each concentration in the range of $69-90 \%$, the mergence of the above formations is observed. In this case, the texture gradually transforms into fan-like texture. 


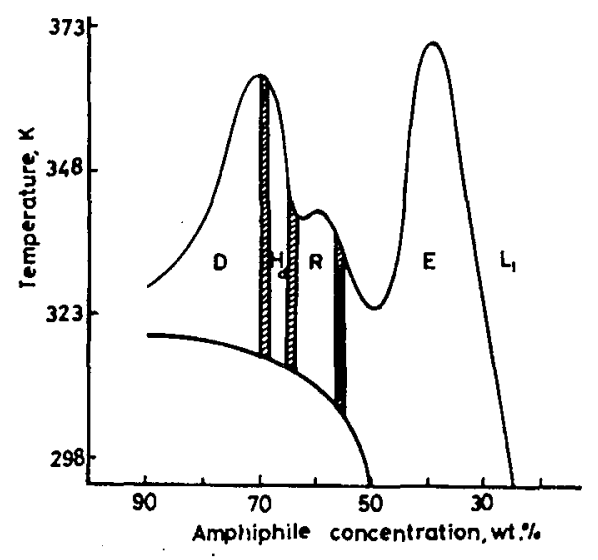

Fig. 2. Phase diagram of potassium oleate+water lyotropic system.

With an amphiphile concentration of $55-90 \%$ and relatively low temperatures (up to $20^{\circ} \mathrm{C}$ ) the system is characterized by a high viscosity and shows a texture corresponding to the gel state. The above texture is a system of separate birefringent and close packed formations. When this texture is heated to the isotropic liquid state followed by cooling up to the gel state, a polycrystalline texture, strongly differing from the basic texture of the gel state, has been observed (Fig. 1e). In the further heating-cooling process of the sample the lyotropic system returns to the state with the above polycrystalline texture again. Identification of the textures according to the type of the observed textures and the character of their transformations with temperature and concentration variations in accordance with the well-known methods of polarization microscopy $[1,17,18]$ allowed to reveal four types of liquid crystalline mesophases in $\mathrm{PO}+$ water system: a simple hexagonal mesophase $E$, a rectangular mesophase $R$, a complex hexagonal mesophase $H_{c}$ and a lamellar mesophase $D$. The temperature-concentration diagram of the phase states of PO+water system is shown in Fig. 2. The shaded regions of the phase diagram represent the concentration phase transition regions among the mesophases.

The investigations of $\mathrm{PO}+$ water+decanol lyotropic system have shown that the above system has a liquid crystalline texture considerably differing from the textures of $\mathrm{PO}+$ water lyotropic system. The analysis of the textures considered has allowed the construction of the phase state diagram of $\mathrm{PO}+$ water+decanol system (Fig. 3). As seen from the diagram, the following mesophases are observed in the lyotropic system studied: a simple hexagonal mesophase $E$, a complex hexagonal mesophase $H_{c}$, a lamellar mesophase $D$, a lamellar mesophase $B$, a rectangular mesophase $R$ and a simple hexagonal mesophase $F$ with inverted micellar aggregates.

The mesophase $E$ was initially characterized by a confocal texture with shallow confocals. In the course of time, this texture transformed into a large-confocal texture (Fig. 4a) well-known from the works on lyotropic mesophase identification 




Fig. 3. Phase diagram of potassium oleate+decanol+water lyotropic system.

$[1,17,18]$. The mesophase $H_{c}$ has a characteristic texture similar to that observed for PO+water system. In the regions of low $\mathrm{DeOH}$ concentrations $(\approx 10 \%)$ the system studied is characterized by a pseudoisotropic texture (Fig. 4b) which can gradually be transformed to a shallow-mosaic texture (Fig. 4c). As it is known, the both types of textures indicate the existence of the layer packing of micellar aggregates. In the range of high concentrations of $\mathrm{DeOH}(\approx 25 \div 27 \%)$ "a petal" texture was observed (Fig. 4d). As seen in Fig. 4d, the elements with optically positive and negative signs (spherulites with light and dark crosses) alternate with one another. As the microscope table rotates, they alternately quenched and brightened.

The mesophase $B$ is characterized by interlaced texture with particular imbedments of regions with a degenerate homeotropic ordering (Fig. 4e). The above mesophase is well oriented by the shift of supporting surfaces of a plane capillary. The shift is responsible for the break of binding between the polycrystalline formations of textures and leads to the formation of a homogeneous orientation.

The peculiarity of the ternary system studied is the existence of two types of layer mesophases, i.e., a lamellar mesophase $D$ with the parallel layers and a lamellar mesophase $B$ with the deformed layers. In this case, the mesophase $D$ is observed in rather wide concentration regions, and this is a distinguishing feature of the given lyotropic system. The mesophase $B$ is observed in a narrow concentration region at low alcohol and amphiphile content (Fig. 3).

The mesophase $F$ with inverted micellar aggregates has a complex confocal texture with confocal formations of different sizes (Fig. 4f). By comparison of Fig. $4 \mathrm{a}$ and Fig. 4f, one can see that the textures of the mesophase $E$ are similar to those of the mesophase $F$. It is clear as the both textures correspond to hexagonal mesophases with the rod-like micellar aggregates. 




(a)



(c)

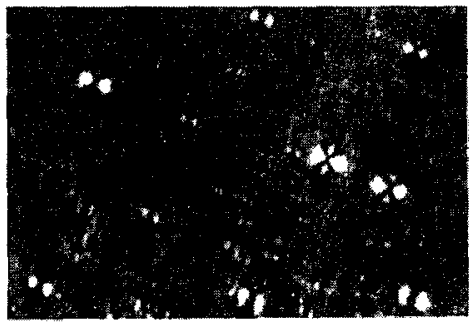

(e)

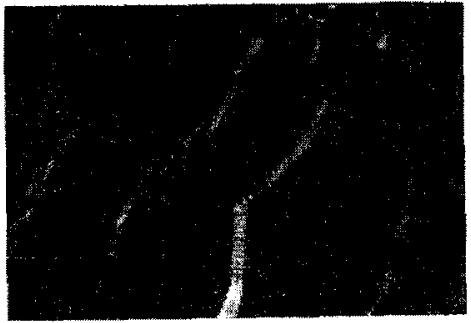

(b)



(d)

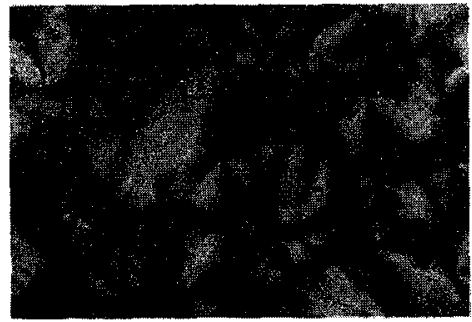

(t)

Fig. 4. Textures of potassium oleate+decanol+water lyotropic system: (a) texture of simple hexagonal mesophase $E$, (b) pseudoisotropic texture of lamellarie mesophase $D$ $(\approx 10 \mathrm{wt} \% \mathrm{DeOH}),(\mathrm{c})$ mosaic texture of lamellare mesophase $(\approx 10 \mathrm{wt} \% \mathrm{DeOH})$, (d) texture of lamellare mesophase $D(\approx 25 \div 27 \mathrm{wt} \% \mathrm{DeOH}),(\mathrm{e})$ texture of lamellare mesophase $B$, (f) texture of hexagonal mesophase $F$ with inverted micellare aggregates.

Taking into account the sequence of alternation of lyotropic phases, namely, the concentration phase transitions "lamellar mesophase $D \rightarrow$ cubic isotropic phase with inverted micellars $I_{2} \rightarrow$ simple hexagonal mesophase with inverted micellars $F^{\prime \prime}$, one can assume the existence of a cubic phase $I_{2}$ with an optical isotropy between the mesophases $D$ and $F$. However, the phase $I_{2}$ was not observed in the system studied. The phase transition "mesophase $D \rightarrow$ mesophase $F$ " was realized through a sufficiently wide concentration region of the coexistence of mesophase $D+F$. It should be noted that the existence of the mesophase $F$ in a rather wide concentration region is a peculiarity of $\mathrm{PO}+$ water+DeOH lyotropic system. In most ternary amphiphile+water+alcohol systems studied this mesophase is either lacking or is revealed in a narrow concentration region. 
At a high alcohol concentration and low water concentrations the isotropic phase was observed. This concentration region corresponds to isotropic micellar phase $L_{2}$ with inverted spherical micellar aggregates. Optically no difference between isotropic phases $L_{1}$ and $L_{2}$ was observed.

The boundaries between lyotropic phases, especially at low alcohol concentrations, were found not to be sharp. In this case, the coexistence of two and even three lyotropic phases was observed. Such behavior of the system points to the presence of the concentration phase transition.

\section{Conclusion}

The comparison of the phase diagrams of $\mathrm{PO}+$ water and $\mathrm{PO}+$ water $+\mathrm{DeOH}$ lyotropic system has shown that at low concentrations of $\mathrm{DeOH}(0-3 \mathrm{wt} \%)$ the ternary lyotropic system has the same phases as the binary PO+water system. With a high concentration of $\mathrm{DeOH}$ the mesophases lacking in the binary system studied occur.

The similar results have also been observed in other lyotropic systems [25-29]. Thus, the addition of alcohol to amphiphile+water lyotropic system leads to the increase in lyotropic polymorphism and to the formation of mesophases unobservable in amphiphile+water system, particularly, to the formation of mesophase with inverted micellar aggregates.

Finally, note that the alcohol addition to an amphiphile+water lyotropic system results in the formation of new characteristic textures. In other words, one and the same lyotropic mesophase in amphiphile +water and in amphiphile+water+alcohol lyotropic system can be characterized by different characteristic textures.

\section{Acknowledgment}

This work was partially supported by Science Foundation of Ege University, grant No. 010/Fen/97.

\section{References}

[1] P. Ekwal, Adv. Liq. Cryst. 1, 1 (1975).

[2] P. Ekwal, L. Mandell, K. Fontell, J. Colloid Interface Sci. 31, 508 (1969).

[3] A. Nesrullajev, F. Rustamov, A. Sonin, Crystallography 29, 1133 (1984).

[4] M.R. Rizzatti, J.D. Gault, J. Colloid Interface Sci. 110, 257 (1986).

[5] G. Bartusch, H.D. Dorfler, H. Hoffmann, Progr. Colloid Polym. Sci. 89, 307 (1992).

[6] R. Bartolino, M. Meuti; G. Chidichimo, G.A. Ranieri, in: Physics of Amph: Micelles, Vesicles and Microemulsions, Eds. V. Degiorgio, M. Corti, North Holland Publ., Amsterdam 1986, p. 524.

[7] S: Göbel, K. Hiltrop, Progr. Colloid Polym. Sci 84, 241 (1991).

[8] F. Mori, J.C. Lim, C.A. Miller, Progr. Colloid Polym. Sci. 82, 114 (1990).

[9] F. Mahmoud, W.S. Higazy, S.D. Chrisrian, E.E. Tucker, A.A. Taha, J. Colloid Interface Sci. 131, 96 (1989).

[10] Th. van den Boomgaard, Sh.M. Zourab, J. Lyklema, Progr. Colloid Polym. Sci. 68, 25 (1983). 
[11] A. Nesrullajew, F.A. Rustamov, Mol. Cryst. Liq. Cryst. 152, 421 (1987).

[12] E. Hecht, Optics, Addison-Wesley Publ. Company, Amsterdam 1987.

[13] N.M. Melankholin, Methods of Investigations of Crystal's Optical Properties, Science Publ., Moscow 1070.

[14] P. de Gennes, Physics of Liquid Crystals, Clarendon Press, Oxford 1974.

[15] D. Demus, H. Demus, H. Zaschke, Flussige Kristalle in Tabellen, VEB Deutscher Verlag Grundstoffindustrie, Leipzig 1976.

[16] D. Demus, L. Richter, Textures of Liquid Crystals, VEB Verlag, Leipzig 1972.

[17] F.B. Rosevear, J. Soc. Cosmet. Chem. 19, 581 (1968).

[18] P. Ekwall, in: Liquid Crystals and Ordered, Fluids, Eds. J.F. Johnson, R.S. Porter, Vol. 2, Plenum Press, New York 1974, p. 177.

[19] G. Hertel, H. Hoffmann, Liquid Cryst. 5, 1883 (1989).

[20] S. Millan, G. Sandaz, L.A. Fucugauchi, Mol. Cryst. Liq. Cryst. 136, 153 (1986).

[21] A. Saupe, J. Colloid Interface Sci. 58, 549 (1976).

[22] F. Grandjean, Compt. Rend. 116, 165 (1917).

[23] G. Friedel, Ann. Phys. 18, 273 (1922).

[24] Plastic Crystalls and Liquid Crystals, Eds. G.W. Gray, P.A. Winsor, Ellis Horwood Publ., Chichester 1975.

[25] T. Kato, M. Iwai, T. Seiniya, J. Colloid Interface Sci. 130, 439 (1989).

[26] A. Attwood, V. Mosquera, V. Perez-Villar, J. Colloid Interface Sci. 127, 532 (1989).

[27] J. Sjöblom, A.M. Blochus, W.M. Sun, S.E. Friebèrg, J. Colloid Interface Sci. 140, 481 (1990).

[28] J. Lang, R. Rueff, M. Dinh-Cao, R. Zana, J. Colloid Interface Sci. 101, 184 (1984).

[29] G. Hertel, Lyotrope nematische Phasen. Der Zusammenhang zwischen Molekülstruktur und Phasenverhalten, Ph.D. Dissertation, Bayreuth 1989. 